\title{
Entorno Personal de Aprendizaje (PLE) basado en objetos virtuales como refuerzo en matemáticas.
}

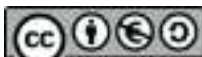

\section{Personal Learning Environment (PLE) based on virtual objects as}

reinforcement in mathematics.

\author{
Diana Nancy Martínez García ${ }^{1}$
}

Recibido: 13-02-2019 / Revisado: 15-02-2019 /Aceptado: 04-03-2019/ Publicado: 04-06-2019

\begin{abstract}
DOI: https://doi.org/10.33262/cienciadigital.v9i2.382

This work aims to develop a personal learning environment to reinforce math with the eighth-year students of basic education; the methodology used was exploratory, because there is not a previous model within an educational model that helps to develop this work. The combination of two types of developmental methodology such as the instructional model to create learning tools are based on the ADDIE model and the ADITE methodology which is a design model to elaborate instructional tools based on computers. The survey was used to extract information, directed to a sample of 30 teachers in 3 different schools of Ambato who were inquired about the integration of TIC resources in their academic practice. The obtained results during the survey implementation showed that there are a few teachers that use technology tools to enhance their teaching practice. Personal learning environment is based on a virtual platform known as Wix, where different tools are combined to create a PLE structure that includes activities related to the first four curricular areas established by the Ministry of Education, in addition of having a module of evaluation activities that contributes to the reinforcement of the mathematics area with specific subjects that allows to enhance the teaching - learning process.
\end{abstract}

Keywords: BLearning, connectivism, virtual platforms, virtual education, Personal Learning Enviroment.

\section{Resumen}

El presente trabajo tiene como finalidad la elaboración de un entorno personal de aprendizaje para el refuerzo de matemáticas en los alumnos de octavo año de educación general básica. La metodología de investigación utilizada es la exploratoria por cuanto no existe un modelo anterior en un contexto educativo propio que sirva de base para el desarrollo del trabajo. Se optó también por una

\footnotetext{
${ }^{1}$ Universidad Técnica de Ambato, Ecuador, dnmartinezg@outlook.com
} 
combinación de dos tipos de metodología de desarrollo como el diseño instruccional para desarrollo de objetos de aprendizaje basado en el modelo ADDIE y la metodología ADITE para la producción de medios instruccionales de carácter informático. La extracción de información se realizó por medio de encuestas, que se aplicaron a una muestra de 30 docentes en 3 unidades educativas de la ciudad de Ambato. En dichas encuestas se indagó sobre la integración de recursos TIC en la práctica académica de los docentes. Los resultados obtenidos evidenciaron que todavía es escaso el número de profesores que utilizan herramientas tecnológicas para potenciar su práctica docente. El PLE o entorno personal de aprendizaje se apoya en una plataforma virtual conocida como Wix en donde se integran diferentes herramientas que permiten armar la estructura del PLE, el cual abarca actividades correspondientes a los cuatro primeros bloques curriculares establecidos por el Ministerio de Educación, además de un módulo de actividades de evaluación que contribuye al refuerzo del área de matemáticas en temas específicos y permite potenciar el proceso de enseñanza - aprendizaje.

Palabras clave: BLearning, conectivismo, plataformas virtuales, educación virtual, Entornos Personales de Aprendizaje.

\section{Introducción.}

En los últimos años se ha observado un vertiginoso interés por aprovechar los innumerables recursos que las TIC ofrecen a los docentes para potenciar el proceso de enseñanza-aprendizaje, así como una amplia apertura de los niños y en la inclusión de estas tecnologías en su desempeño académico y otros ámbitos de sus vidas (Litwin, 2005).

No se puede asegurar que la reciente inserción de las nuevas tecnologías se convierta en la panacea de todos los problemas académicos del sistema educativo, pero al permitir mayor autonomía en el proceso de aprendizaje y en la gestión del conocimiento; la construcción de nuevo conocimiento y la disponibilidad de diversas fuentes de información, responde a dos pilares fundamentales de la educación del siglo XXI: “aprender a aprender" y “aprender a hacer" (Delors, 1996).

En las búsquedas bibliográficas llevadas a cabo no se han detectado diseños de PLE por parte de docentes, lo que implica un desconocimiento de este recurso como estrategia didáctica para la formación. El material educativo empleado por los docentes, en forma general, se presenta como documentos estáticos que no motivan el interés de los estudiantes por aprender.

Este proyecto pretende ser una herramienta que potencie la experiencia del aprendizaje abarcando los bloques curriculares de números enteros, números decimales, números fraccionarios e iniciación al álgebra por medio de la compilación de material didáctico de la Web que cumpla con los objetivos requeridos por parte de currículo de educación general básica, incluyendo también un módulo de ejercicios de autoevaluación que permitan complementar el refuerzo académico del área de matemáticas. 


\section{Metodología.}

El desarrollo propuesto se elaboró en ambiente web, en un entorno virtual conocido como Wix, en el cual se integran diferentes objetos virtuales de aprendizaje como aplicaciones multimedia con YouTube, software educativo como Cuadernia, Daypo, marcadores sociales como SymbalooEDU, recursos para obtener información como motores de búsqueda Google, herramientas colaborativas como repositorios Google Docs y redes sociales como Facebook y Twitter.

En esta etapa se realizó la socialización del producto final a 30 docentes de matemáticas de diferentes instituciones educativas de la ciudad de Ambato. Para la ejecución de esta etapa se solicitó la autorización a las diferentes autoridades de las instituciones educativas, y la colaboración del director del Distrito Ambato $\mathrm{N}^{\circ} 1$, lo que permitió contar con la presencia de varias instituciones pertenecientes a dicho distrito.

Para medir el impacto del proyecto se utilizó el cuestionario como instrumento de evaluación, el cual contenía 5 indicadores cuyo principal objetivo fue determinar la opinión de los docentes que utilizaron el producto final en las categorías de presentación, navegabilidad, funcionamiento, pertinencia e integración en la práctica docente. La siguiente tabla muestra el instrumento de evaluación preliminar utilizado para medir el impacto del producto final.

\section{Diagnóstico.}

El mecanismo empleado para la extracción de la información fue el cuestionario mediante el cual se indagó sobre diferentes aspectos del uso de TIC y competencias digitales en la práctica docente.

Los temas que se tomaron en consideración para la elaboración del instrumento están estrechamente relacionados con el tema de desarrollo planteado:

- Innovación educativa con contenido y material didáctico digital.

- Ambientes virtuales para enseñanza y aprendizaje.

- Aprendizaje autónomo y trabajo colaborativo.

- Comunicación e interacción con medios electrónicos.

\section{Métodos Aplicados}

\section{De investigación}

\section{Investigación exploratoria.}

Para la realización del presente proyecto se empleó el tipo de investigación exploratoria por cuanto no existe un modelo anterior, en un contexto educativo propio, que sirve de 
base para el desarrollo del trabajo.

\section{Técnicas.}

La técnica empleada para la recolección de la información fue la encuesta, la cual constaba de nueve preguntas que indagaban acerca de diferentes aspectos sobre el uso de TIC en la práctica docente, la misma que fue aplicada a un número determinado de profesores de distintas áreas y niveles educativos, así como de diferentes unidades educativas de la ciudad de Ambato.

\section{Resultados.}

Figura 1. Número de docentes por área y niveles.

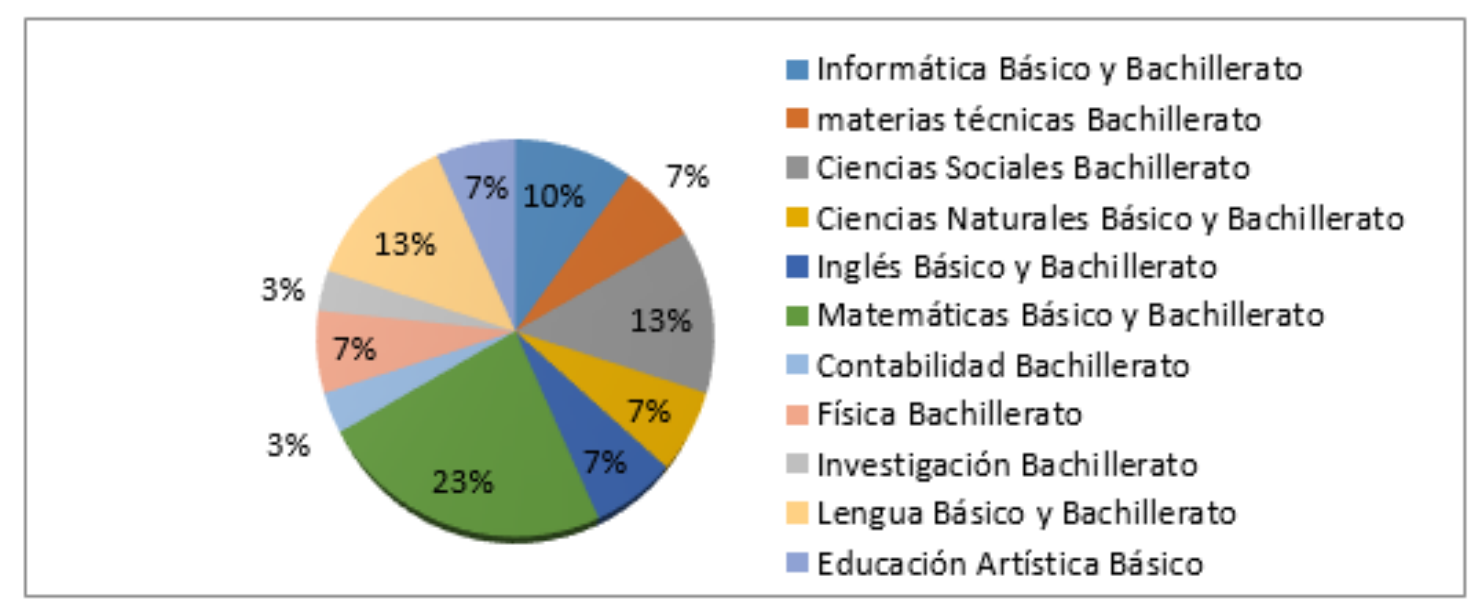

La figura 1 muestra que, del número total de docentes encuestados, el $23 \%$ corresponde al área de Matemáticas en los niveles de educación general básica y bachillerato, el $13 \%$ es del área de Ciencias Sociales y de Lengua y el 7 \% corresponde a docentes de áreas de materias técnicas, inglés, Ciencias Naturales, Educación Artística y Física. Se observa que el mayor porcentaje corresponde a docentes del área de matemáticas que es el grupo hacia el cual está dirigido este desarrollo. 
Figura 2: Nivel de conocimientos entornos y herramientas informáticas.

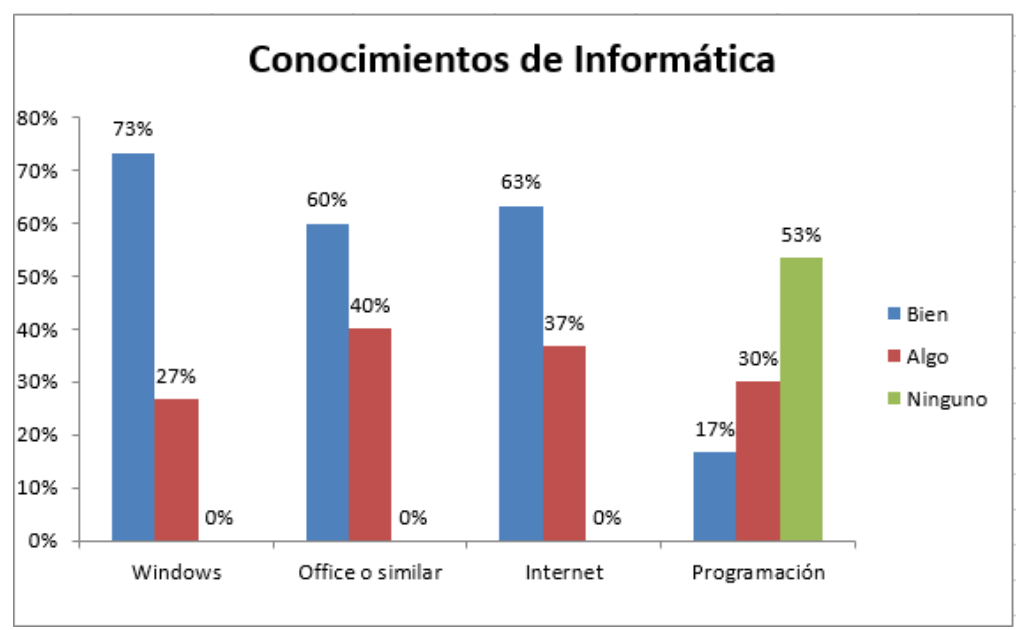

En esta figura se evidencia que la mayoría de docentes, lo que corresponde al $73 \%$ conoce el entorno de Windows, pero este número se reduce cuando el tipo de aplicaciones se especifican. En el caso de Internet, 19 de 30 docentes está bien familiarizados con su manejo. En cuanto a uso de Office, solamente 18 de 30 lo conocen bien. En lo que respecta a conocimientos en programación sólo el 17 \% tiene conocimientos profundos del área lo que indica que este grupo de docentes son especialista en dicho campo mientras que el resto de docentes encuestados pertenece a áreas ajenas al campo de la tecnología. De este análisis se puede concluir que la mayoría de los docentes conoce lo básico para poder desenvolverse en actividades de enseñanza.

Figura 3: Aspectos básicos del uso de TIC.

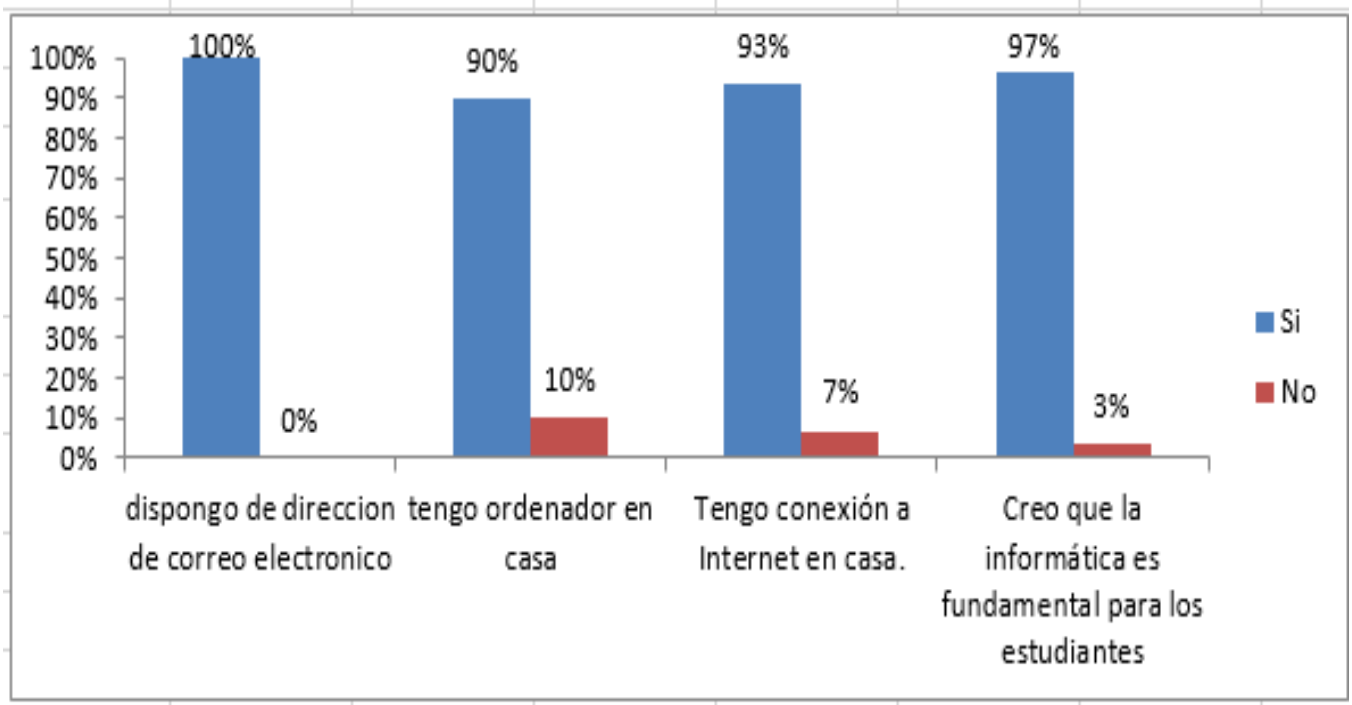

Dentro de este aspecto se observa que, en los cuatro ítems evaluados, los docentes se han integrado al uso de nuevas tecnologías en distintos ámbitos de su vida además del laboral, dando a notar la reducción de la brecha digital, permitiendo mayor acceso a las TIC, considerando como fundamental la integración de estas en el ámbito educativo 
Figura 4: Integración de los docentes en procesos de formación virtual.

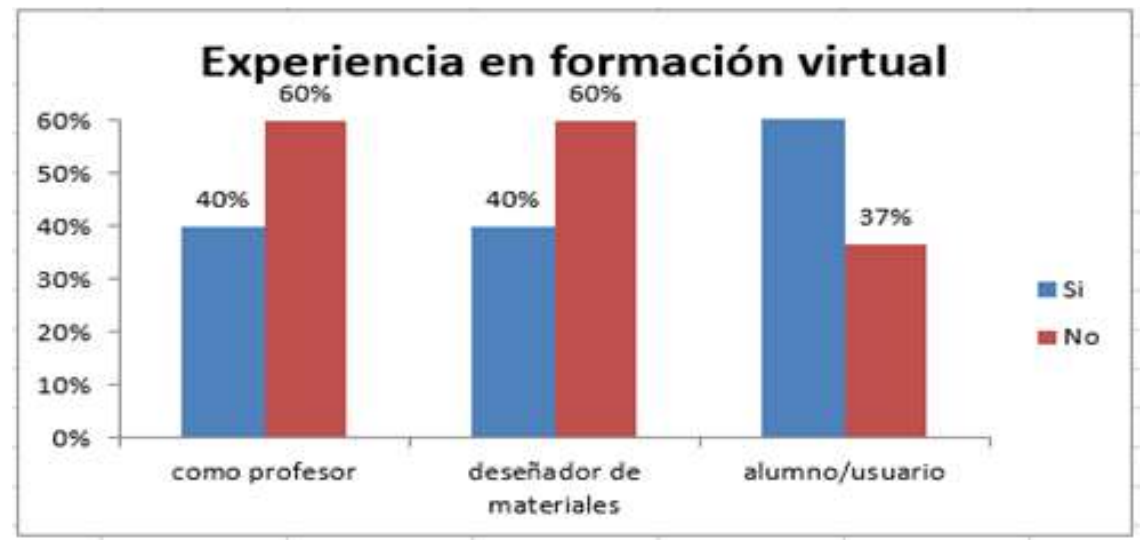

La figura 4, permite observar que la mayoría de docentes se adaptan a los nuevos procesos formativos y que además están incursionando en diferentes aspectos del mismo, asumiendo los roles de profesor como de estudiante, aunque en este último se observa un ligero aumento, se evidencia que existe interés en nuevas formas de aprender.

Figura 5: Uso de software educativo relacionado con la asignatura que imparte

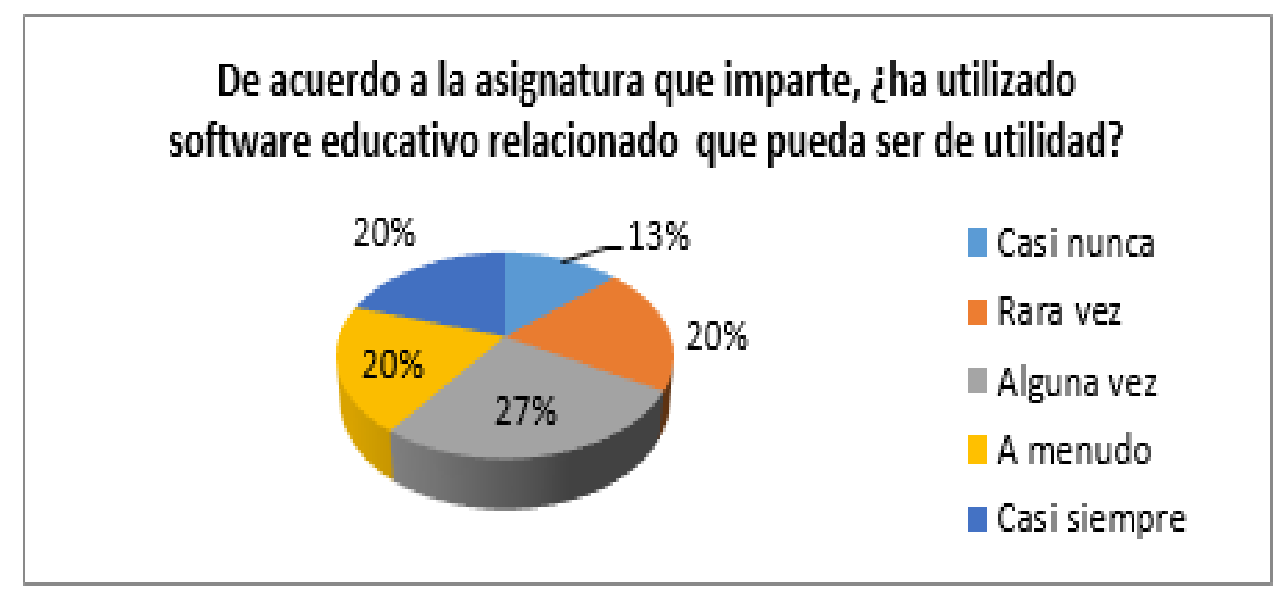

La figura 5 evidencia que el uso de software educativo relacionado con la asignatura que cada profesor imparte es un recurso que se utiliza en distintas ocasiones; en forma mayoritaria por el $27 \%$ de los docentes encuestados, mientras que un grupo que corresponde al $20 \%$ lo usa casi siempre, a menudo, y con menor frecuencia respectivamente. Finalmente, el $13 \%$ casi nunca integra este tipo de herramientas a su práctica docente. 
Figura 6: Uso de recursos educativos de Internet.

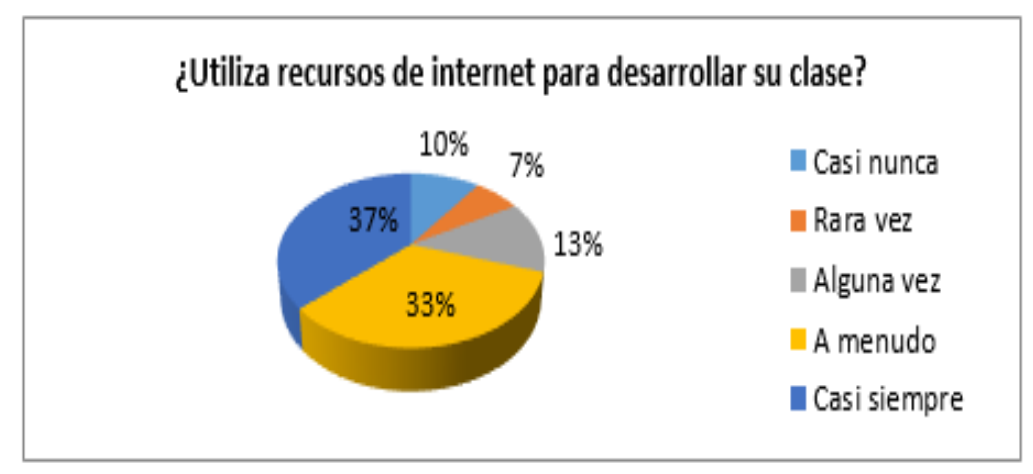

La tendencia al uso de internet para la práctica docente se evidencia en este gráfico el cual permite observar que la mayoría de los docentes encuestados en un porcentaje del $37 \%$ y $33 \%$ en cuanto a frecuencia de uso se refiere, opta por el aprovechamiento de recursos pre elaborados para integrar en sus asignaturas, aunque mucha de la información que circula en internet no necesariamente es de calidad, muchos de estos recursos si son útiles y así lo demuestra la preferencia de los docentes.

Figura 7. Ambientes virtuales de aprendizaje.

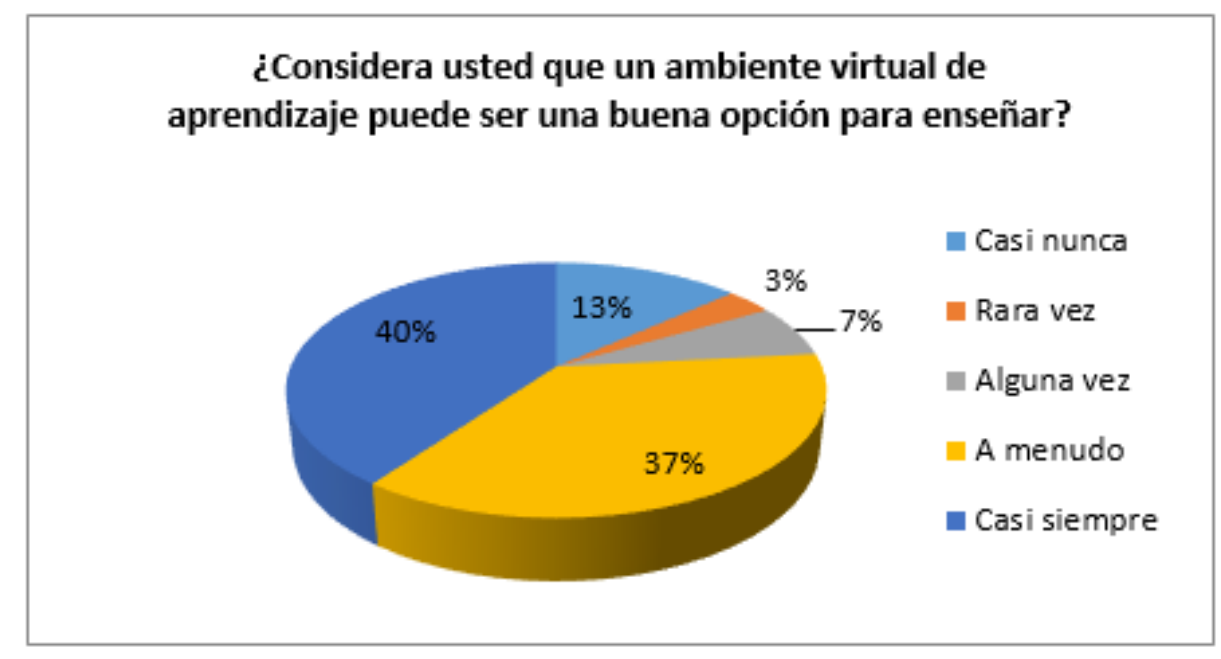

El $40 \%$ de los docentes encuestados considera los ambientes virtuales de aprendizaje como una buena opción a integrar en el proceso de enseñanza - aprendizaje, mientras que el $3 \%$ del mismo grupo no lo cree de esta manera, por lo tanto, su frecuencia de uso de este tipo de recursos es bastante baja. 
Figura 8: Las TIC potenciadora de la labor docente

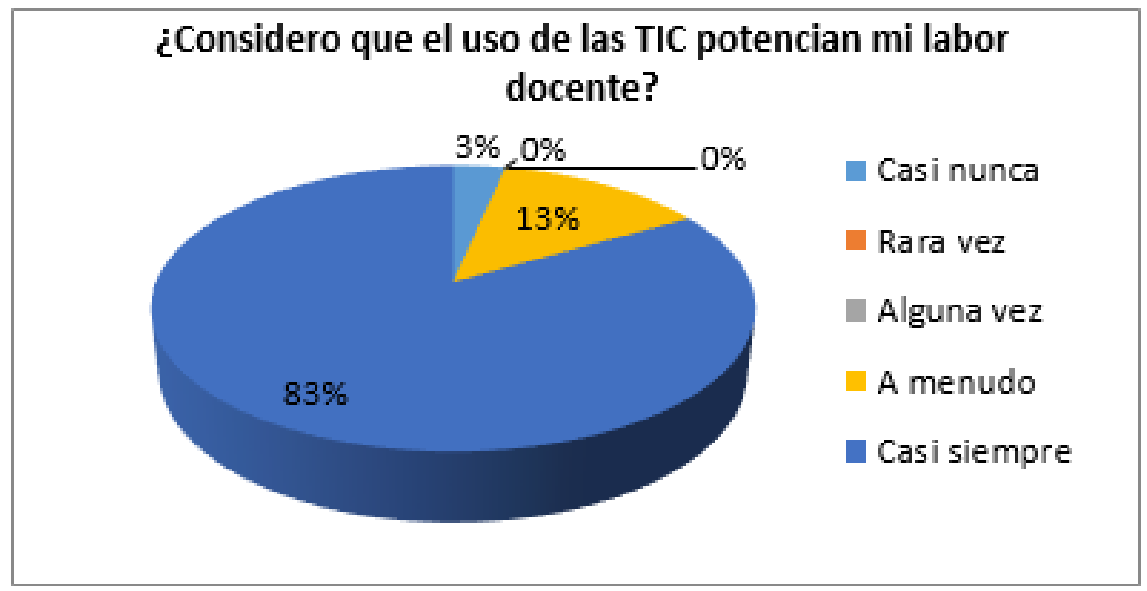

Esta figura representa la aceptación de los docentes al uso de herramientas tecnológicas como apoyo a sus prácticas. Un $83 \%$ estima que le son de utilidad considerando la integración de las TIC como herramientas potenciadoras para su labor docente; un $13 \%$ considera que, a menudo, esta premisa es valedera, mientras que un $3 \%$ de los maestros opina que el uso de TIC no es relevante en su práctica docente.

\section{Conclusiones.}

- El PLE (entorno personal de aprendizaje) y los OVAs (objetos virtuales de aprendizaje) fomentan un proceso formativo más flexible en donde se integran y desarrollan nuevas habilidades al permitir mayor autonomía en la gestión, construcción de nuevo conocimiento a través de la disponibilidad de diversas fuentes de información.

- Se diagnosticó sobre el uso de TIC en la práctica docente en los profesores de diferentes unidades educativas de la ciudad de Ambato. El análisis del instrumento de diagnóstico aplicado evidenció que los maestros de la ciudad de Ambato tienen predisposición para integrar las tecnologías en sus prácticas docentes.

- Para la elaboración del PLE y los OVAs se implementó una combinación de dos metodologías orientadas a la elaboración de objetos virtuales de aprendizaje, ADDIE y ADITE, las cuales se complementaron para permitir coordinar de manera lógica la elaboración de la propuesta.

\section{Referencias Bibliográficas}

Canellas Cabrera, A. M. (19 de Julio de 2006). Quaderns Digitals. Recuperado el 13 de Abril de 2014, de Hemeroteca virtual: http://quadernsdigitals.net/index.php?accionMenu=hemeroteca.VisualizaArticul oIU.visualiza\&articulo_id=9250

Cuenca, R. T., Hernández, L. G. Á., \& Márquez, N. D. C. Á. (2018). INDICADORES PARA LA EVALUACIÓN DEL USO DE RECURSOS VIRTUALES DE 
APRENDIZAJE EN LA UNIVERSIDAD DE HOLGUÍN. REFCalE: Revista Electrónica Formación y Calidad Educativa. ISSN 1390-9010, 6(2), 69-84.

Del Moral, M., Cernea, D., \& Lourdes, V. (2010). RED: Revista de Edcucación a distancia. Obtenido de OBJETOS DE APRENDIZAJE 2.0 UNA NUEVA GENERACIÓN DE CONTENIDOS EN CONTEXTOS CONECTIVISTAS: http://www.um.es/ead/red/25/esther.pdf

Delors, J. (1996). Los cinco pilares de la educación. En J. Delors, La educación encierra un tesoro. (pág. 35). UNESCO.

Mestre, U., Fonseca, J. J., \& Valdés, P. (2007). Libros Digitales. (E. Universitaria, Ed.) Recuperado el Abril de 2014, de ENTORNOS VIRTUALES DE ENSEÑANZA - APRENDIZAJE: http://www.librosdigitales.org/bitstream/001/251/8/978-95916-0637-2.pdf

Ministerio de Educación del Ecuador. (2010). El perfil de salida de los estudiantes de educación general básica. En M. d. Ecuador, Actualización y Fortalecimiento curricular de Educación General Básica (pág. 14). Quito, Pichincha, Ecuador.

Ministerio de Educación del Ecuador. (2010). Indicadors esenciales de evaluación. En M. d. Ecuador, Actualización y Fortalecimiento curricular de educación general básica (pág. 41). Quito, Pichincha, Ecuador.

Ministerio de Educación del Ecuador. (2010). La importancia de enseñar y aprender matemática. En M. d. Ecuador, Actualización y Fortalecimiento curricular de Educción General Básica (pág. 23). Quito, Pichincha, Ecuador.

Ministerio de Educación del Ecuador. (2010). Objetivos Educativos del año. En M. d. Educación, Actualización y Fortalecimiento curricular de edcuación generral básica (pág. 30). Quito, Pichincha, Ecuador.

Naranjo, Y. V. J., \& Osuna, J. B. (2017). Aproximación al análisis de los entornos personales de aprendizaje de los estudiantes de la Universidad Tecnológica de La Habana, CUJAE. In Innovación docente y uso de las TIC en eduación: CD-ROM (p. 21). Universidad de Málaga (UMA).

Polo, M. (2003). Aproximación a un modelo de diseño: ADITE. Recuperado el 1 de Marzo de 2015, de Universidad Central de Venezuela: http://www.ucv.ve/fileadmin/user_upload/sadpro/Documentos/docencia_vol4_n 1_2003/7_art._4Marina_Polo.pdf

Sánchez Medina, I. (2014). Estado del arte de las metodologías y modelos de los Objetos Virtuales de Aprendizaje (OVAS) en Colombia. Entornos, (28), 93-107. 
https://doi.org/10.25054/01247905.528

Segura, J., \& Quintero, L. (2010). Digitum. Recuperado el Abril de 2014, de Los Entornos Personales de Aprendizaje (PLEs): una nueva manera de entender el aprendizaje: http://digitum.um.es/jspui/bitstream/10201/17247/1/Adell\%26Casta\%C3\%B1ed a_2010.pdf

Wiley, D. A. (2000). Reusability. Recuperado el 31 de Mayo de 2014, de Connecting learning objects to instructional design theory: A definition, a metaphor, and a taxonomy: http://reusability.org/read/chapters/wiley.doc 


\section{Para citar el artículo indexado.}

Martínez D. (2019). Entorno personal de aprendizaje (PLE) basado en objetos virtuales como refuerzo en matemáticas. Revista electrónica Ciencia Digital 3(2), 161-171. Recuperado desde: http://cienciadigital.org/revistacienciadigital2/index.php/CienciaDigital/article/view/382 $\underline{1830}$

\section{\Ciencia}

El artículo que se publica es de exclusiva responsabilidad de los autores y no necesariamente reflejan el pensamiento de la Revista Ciencia Digital.

El artículo queda en propiedad de la revista y, por tanto, su publicación parcial y/o total en otro medio tiene que ser autorizado por el director de la Revista Ciencia Digital.
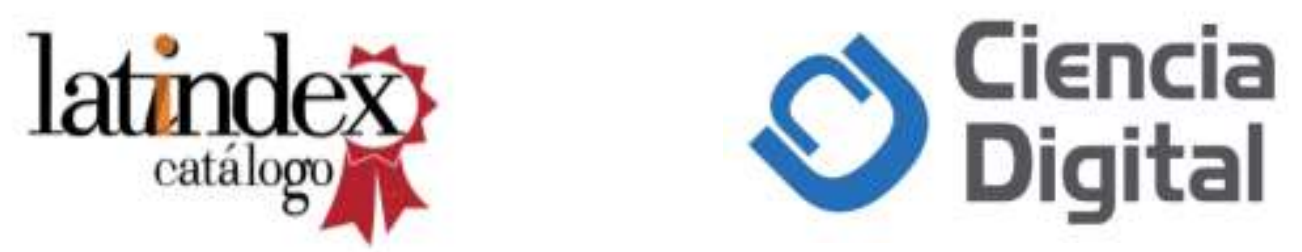